\title{
Sleep pattern, common bedtime problems, and related factors among first- grade students: Epidemiology and predictors
}

\author{
Habibolah Khazaie $^{\mathrm{a}}$, Ali Zakiei ${ }^{\mathrm{a}, *}$, Mohsen Rezaei $^{\mathrm{a}}$, Saeid Komasi ${ }^{\mathrm{b}}$, Serge Brand ${ }^{\mathrm{a}, \mathrm{c}, \mathrm{d}, \mathrm{e}}$ \\ ${ }^{a}$ Sleep Disorders Research Center, Kermanshah University of Medical Sciences, Kermanshah, Iran \\ ${ }^{\mathrm{b}}$ Clinical Research Development Center, Imam Reza Hospital, Kermanshah University of Medical Sciences, Kermanshah, Iran \\ ${ }^{c}$ Substance Abuse Prevention Research Center, Kermanshah University of Medical Sciences, Kermanshah, Iran \\ ${ }^{\mathrm{d}}$ Psychiatric Clinics (UPK), Center for Affective, Stress and Sleep Disorders (ZASS), University of Basel, Basel, Switzerland \\ ${ }^{\mathrm{e}}$ Department of Sport, Exercise and Health, Division of Sport Science and Psychosocial Health, University of Basel, Basel, Switzerland
}

A R T I C L E I N F O

\section{Keywords:}

Children

School

Sleep

Sleep hygiene

\begin{abstract}
A B S T R A C T
Objectives: Investigating sleep patterns and prevalence of sleep problems among children, particularly at the first year of school, is the main objective of the current study. After evaluating sleep pattern and common bedtime problems among children, a number of factors related to these issues were also addressed.

Methods: The statistical population of the cross-sectional study includes all the first-grade students of elementary schools around Kermanshah City in western Iran, among which a sample of 644 participants were selected. The sampling method was a multi-step clustering method and the obtained data were analyzed using Pearson's correlation and binary logistic regression analyze.

Results: The results of the study show that $30.7 \%$ of the participating children suffer from resistance to falling asleep, $15.8 \%$ suffer from resistance to waking up from sleep, 13.9\% suffer from waking up during sleep, $26.1 \%$ suffer from insufficient sleep, and finally $11.2 \%$ suffer from sleep-disordered breathing. Accordingly, sleep quality among $7.6 \%$ of the children was poor; this factor was $5.1 \%$ among girls and $10.3 \%$ among boys $(\mathrm{P}<0.01)$. Moreover, there was a significant relationship between sleep hygiene and sleep problems $(\mathrm{P}<0.001)$. Especially, components of planning/worrying $(\mathrm{OR}=0.49-1.84)$ and exercise $(\mathrm{OR}=0.73-1.34)$ are main predictors of sleep problems.

Conclusions: Based on the results of the study, it can be said that $57 \%$ of the first-grade students suffer from at least one sleep problem. Poor sleep hygiene is an important predictor for sleep problem in the first-grade students.
\end{abstract}

\section{Introduction}

Healthy sleeping is an essential component for the natural development of a child. Moreover, it is highly important for the general health and the development of learning skills in school. ${ }^{1,2}$ Research on children shows that the prevalence of sleep problems is between 14 and $45 \%$. ${ }^{3,4}$ Sleep problems reported among children generally include behavioral sleeping problems, problems with falling asleep, waking up during the night, parasomnia, and symptoms related to sleep-disordered breathing. ${ }^{5,6}$ Recent studies show that sleep problems are highly prevalent among children. ${ }^{7-9}$ Since sleep problems among children are often related to psychological complications and illnesses, identifying related factors are of utmost importance. ${ }^{10-12}$ It is argued that sleep problems during childhood are related to mental health, ${ }^{13}$ and physical and bodily health. ${ }^{14}$ Furthermore, some studies show that sleep problems in children are related to emotional problems such as anxiety and depression in later stages of their lives. ${ }^{15-17}$

Many social and demographic factors influence sleep disorders such as the education level of parents, living in urban areas, and lack of sufficient hygiene. ${ }^{18}$ Socioeconomic status, side-effects of some drugs, psychological factors, environmental factors, and the interactions between children and parents can also affect sleep. ${ }^{19}$ Parents' attention to the schoolchild's sleep plays an important role in this regard. ${ }^{11}$ This is referred to as "sleep hygiene", which is considered in the current study.

An undeniable fact is that sleep pattern changes when we age. Generally, the mechanism for the start and continuation of sleep has different patterns from infancy to adulthood. It is believed that there is a reverse relationship between sleep efficiency (quality of sleep) and aging. The effect of age on sleep patterns is a complex process, which cannot be readily understood. Assessing sleep characteristics during life

\footnotetext{
* Corresponding author. Sleep Disorders Research Center, Kermanshah University of Medical Sciences, Kermanshah, Iran.

E-mail address: zakieiali@gmail.com (A. Zakiei).
} 
can contribute in this regard and increase our understanding of the functions of sleep and how to improve sleep quality. ${ }^{20}$

Given the roles the children will play in future society, paying attention to their issues and complications, particularly their sleep quality, are an inevitable necessity. By studying sleep quality among children we can acquire more knowledge in this regard and by identifying the factors affecting it we can obtain strategies to help better improve their sleep quality. On the other hand, the first year of school can affect the children's sleep patterns due to the new conditions it creates for these children. Therefore, the current study aims to evaluate sleep patterns, prevalence of common sleep problems, and the related factors among first-grade students.

\section{Materials and methods}

\subsection{Design, participants, and data collection}

The current study was a cross-sectional one utilizing survey method by using questionnaires. The statistical population of the study included all the male and female firs-grade students of public elementary schools in Kermanshah City in 2017. The sample size was determined to be 322 participants using Cochran's Formula. However, by considering factors such as participant influx, lack of cooperation, and increased accuracy, the sample size was doubled and in general, 644 participants were selected. Finally, the data for 606 participants were analyzed. The sample of the study was selected using multi-step sampling method. In order to select the participants, first we considered each one of the three zones of the Organization for Education and Training of Kermanshah City as a distinct cluster. Then, among the schools in each zone, we selected two female and two male schools. The sample size was distributed among the schools based on the population of the students and gender proportions were also followed for selecting the participants.

The entrance criteria for the study included items such as being free of any chronic or underlying psychological illness and not using any psychoactive drugs, which was determined based on parents' reports and the student health documents. Moreover, the students had to be local to Kermanshah City and their parents had to be still together.

The data were gathered two weeks after the education year started and this process continued for a month. The data gathering process started after acquiring the necessary permits from related organizations. The researcher would go to the schools, contact the mothers of the students and after their consent to participate in the study, the questionnaire would be distributed among them. After getting consents from the parents of participating students, we ensured them that their responses would remain confidential and only be used for research purposes.

\subsection{Instruments}

Questionnaire for Evaluating Sleep Disorders in Children (BEARS): This questionnaire was developed in School of Medicine in Brown University. Its objective is to evaluate sleep problems among kindergarten and elementary school children based on five dimensions. These dimensions include bedtime problems (B), excessive daytime sleepiness (E), awakening during the night (A), regularity and duration of sleep $(\mathrm{R})$, and sleep-disordered breathing (S). ${ }^{21}$
Detailed Sleep Evaluation Questionnaire (DSEQ): This questionnaire was used to gather more details regarding sleep problems of children. Responses to BEARS and DSEQ are yes or no responses and the parents will answer the questions based on their observations with regards to the behaviors and sleep patterns of their children. ${ }^{21}$

Sleep Hygiene Index (SHI): Sleep hygiene index (SHI) is a 13-item measure for evaluating environmental and behavioral variables which can increase unhealthy sleep. This measure was developed in 2006 by Mastin et al. In this questionnaire, each question is scored based on a five-option spectrum (always, repeatedly, sometimes, seldom, never). Researchers calculated a Cronbach's Alpha Coefficient of 0.66 for this questionnaire and a coefficient of 0.71 for the retest. Moreover, the correlation between this index and the Eporth Sleepiness Measure was reported as 0.24 and between this index and Pittsburg Sleep Measure was reported as $0.48 .^{22}$ This questionnaire has been standardized in Iran and its internal consistency has been reported as 0.83 . Also, three factors including sleep-wake cycle behaviors, bedroom factors, and behaviors affecting sleep have been extracted from it. The results for the standardization in Iran show that the SHI has a significant correlation with Pittsburg Sleep Quality Questionnaire, Eporth Sleepiness Questionnaire, and Insomnia Intensity Index. ${ }^{23}$ It is worth mentioning that in this study, the parents filled out the questionnaires; however, the questions were changed so that their child would be the person evaluated. Cronbach's Alpha Coefficient for the Sleep Hygiene Index (SHI) in this study was obtained as 0.89 .

\subsection{Data analysis}

In order to analyze the obtained data, SPSS-21 software application was used. Measures such as means and standard deviation were used for describing the selected variables, chi-square test was used for comparing group frequencies, Pearson's correlation test was used for evaluating the relationship between the variables, and non-adjusted binary logistic regression was used for predicting the dimensions of sleep problems based on sleep hygiene items. The odd ratio for $95 \%$ confidence interval and significant levels were reported as $P<0.05$.

\section{Results}

The sample of the study included 606 participants, among which 312 participants (51.5\%) were girls and 290 participants (47.9\%) were boys, the gender of four participants were unknown (missing data). The average age of the study sample was $6.72 \pm 0.58$. In the sample of mothers, 10 people (1.7\%) were illiterate, 86 people (14.2\%) had under diploma education, 252 people (41.6\%) had a diploma, and 242 people (39.9\%) had a college degree. In the case of fathers, 4 people $(0.7 \%)$ were illiterate, 50 people (8.3\%) had under diploma education, 228 people (37.6\%) had a diploma, and 308 people (50.8\%) had a college degree. The status of education was unknown in the remaining cases. Table 1 presents the frequency and percentage of various dimensions of sleep problems based on gender.

The results in Table 1 show that in our sample, $30.7 \%$ of the participants have the problem of resistance to falling asleep and regarding this variable there is no significant difference between boys and girls students. Moreover, $15.8 \%$ of the participants have difficulty waking up and $13.9 \%$ of them wake up during the night; with regards to this

Table 1

Comparison of percentages of sleep problems in each domain of BEARS questionnaire among boy and girl groups.

\begin{tabular}{|c|c|c|c|c|c|c|}
\hline Components of BEARS & Total & Girls & Boys & $P$-value & In Tehran & In USA \\
\hline Bedtime problems & $186(30.7)$ & $86(27.6)$ & $100(34.5)$ & 0.060 & 21.05 & 16.3 \\
\hline Excessive daytime sleepiness & $96(15.8)$ & $44(14.1)$ & $52(17.9)$ & 0.210 & 42.98 & 5.6 \\
\hline Awakenings during the night & 84 (13.9) & $42(13.5)$ & $42(14.5)$ & 0.789 & 32.46 & 18.4 \\
\hline Regularity and duration of sleep & $158(26.1)$ & $64(20.5)$ & $94(32.4)$ & 0.001 & 17.54 & 5.7 \\
\hline Sleep-disordered breathing & $68(11.2)$ & $34(10.9)$ & 34 (11.7) & 0.690 & 10.53 & 13.1 \\
\hline
\end{tabular}


Table 2

Frequency and percentages of general sleep history and general sleep problem.

\begin{tabular}{|c|c|c|c|c|}
\hline General sleep history & Total & Girls & Boys & $P$-value \\
\hline Child takes a nap & $340(56.1)$ & $178(57.1)$ & $160(55.2)$ & 0.716 \\
\hline Does the child have a regular bedtime routine? & $312(51.5)$ & $152(48.7)$ & $158(54.5)$ & 0.178 \\
\hline Does the child have his/her own bedroom? & $370(61.1)$ & $202(64.7)$ & $166(57.2)$ & 0.074 \\
\hline Does the child have his/her own bed? & $524(86.5)$ & $284(91)$ & $236(81.4)$ & 0.001 \\
\hline Is a parent present when your child falls asleep? & $358(59.1)$ & $196(62.8)$ & $158(54.5)$ & 0.048 \\
\hline General sleep problem & Total & Girls & Boys & $P$-value \\
\hline Child resists going to bed? & $214(35.3)$ & $98(31.4)$ & $116(40)$ & 0.031 \\
\hline Child has difficulty falling asleep? & $50(8.3)$ & $18(5.8)$ & $32(11)$ & 0.019 \\
\hline Child awakens during the night? & $96(15.8)$ & $46(14.7)$ & $50(17.2)$ & 0.399 \\
\hline After nighttime awakening, child has difficulty falling back to sleep? & $26(4.3)$ & $12(3.8)$ & $14(4.8)$ & 0.575 \\
\hline Child is difficult to awaken in the morning? & $132(21.8)$ & $64(20.5)$ & $68(23.4)$ & 0.380 \\
\hline Child is a poor sleeper? & $46(7.6)$ & $16(5.1)$ & $30(10.3)$ & 0.015 \\
\hline
\end{tabular}

variable there is no significant difference between boys and girls students. However, the results show that with regards to "insufficient sleep", reported by $26.1 \%$ of participants, there is a significant difference between boys and girls students; $20.5 \%$ of the girl's participants don't have sufficient sleep while $32.4 \%$ of boy's participants have this problem. On the other hand, the results show that $11.2 \%$ of the participants have sleep-disordered breathing and snoring problems; there was no significant difference between boys and girls participants in this regard. As can be seen from Table 1, "resistance to falling asleep" is more prevalent than other problems among the participants. Generally, the results show that about $57 \%$ of the participants have at least one of the sleep difficulties considered in the study.

The results in Table 2 show that $56.1 \%$ of the participants nap during the day, $51.5 \%$ has a pre-determined time for going to bed, and $61.1 \%$ of the participants have their own bedrooms, with no significant difference between boys and girls students. Moreover, the results show that $86.5 \%$ of the participants have their own bed sheets; $91 \%$ of the girls and $81.4 \%$ of the boys. Furthermore, in $59.1 \%$ of cases, when the children are sleep, the parents stay awake. $15.8 \%$ of the participants report that their children wake up during the night and $21.8 \%$ of them reported that their children have difficulty getting out of bed in the morning. Generally, the results show that for $7.6 \%$ of the participants, sleep quality is low, which is $5.1 \%$ among the girls and $10.3 \%$ among the boys, which means a higher percentage of boys have lower sleep quality $(P<0.01)$. The frequency and prevalence percentage of sleep problems at the present and the daily performance of the participants are presented in Table 3 based on gender. In addition, Table 4 shows the means and standard deviation at general sleep history.

Results in Table 5 show that there is a significant negative relationship between parents' education level and the selected variables (except for bedtime problems). Moreover, there is a significant relationship between sleep hygiene and the selected variables (except for general history). In order to predict the dimensions of sleep problems based on sleep hygiene, logistic regression was used and the results of the analysis are presented in Table 6.

The results of binary logistic regression, depicted in Table 6, show that for the dimension of "bedtime problems", the components of sleep hygiene all together can predict $18-25 \%$ of the variance. Evaluating individual sleep hygiene components shows that computer games $[\mathrm{OR}=0.56(0.44-0.72)]$ and complaining and worrying $[\mathrm{OR}=0.49$ $(0.40-0.61)]$ can predict "bedtime problems". For the dimension of "excessive daytime sleepiness", all the sleep hygiene components together can predict $12-20 \%$ of the variance and evaluating the components shows that taking naps [OR $=1.45(1.08-1.94)]$, going to bed at different times $[\mathrm{OR}=0.61 \quad(0.43-0.88)]$, exercise $[\mathrm{OR}=1.29$ (0.98-1.71)], playing video games $[\mathrm{OR}=0.54(0.41-0.72)]$, and complaining and worrying $[\mathrm{OR}=0.75(0.58-0.98)]$ can predict this variable. Moreover, the results show that sleep hygiene components can predict $11-19 \%$ of variance in "awakening during the night", $21-31 \%$ of the variance in "regularity and duration of sleep", and 5-10\% of the variance in "sleep-disordered breathing". Other ORs are visible in Table 6.

\section{Discussion}

The results of our study show that $30.7 \%$ of the participating children have the problem of resistance to falling asleep, $15.8 \%$ have difficulty waking up in the morning, $13.9 \%$ wake up during the night, $26.1 \%$ of them don't have sufficient sleep, and $11.2 \%$ suffer from sleepdisordered breathing and snoring. In fact, "resistance to falling sleep" was more prevalent than other problems among the participants. Generally, based on parents' reports, $7.6 \%$ of the children have low sleep quality, $5.1 \%$ among girls and $10.3 \%$ among boys, which shows that a higher percentage of boys have lower sleep quality. Compared to similar studies on "bedtime problems", $21.05 \%$ of school children in Tehran had this problem, and $16.6 \%$ of the school children in the U.S. had the problem. With regards to "excessive daytime sleepiness", the prevalence rate in the Tehran study was $32.46 \%$ and in the U.S. study, it was $5.6 \%$. With regards to "regularity and duration of sleep", the prevalence rate in the Tehran study was $17.54 \%$ and in the U.S. study, it was $5.7 \%$. With regards to "sleep-disordered breathing", the prevalence rate in the Tehran study was $10.53 \%$ and in the U.S. study, it was $13.1 \% .^{21,24}$

Sleep problems among school age children is a common complication in a way that various studies report its prevalence to be between 20 and $45 \%{ }^{25-28}$ This is while based on our study; about $57 \%$ of the children are suffering from at least one of the sleep problems. While patterns of insufficient sleep and sleep disorders during childhood vary in different cultures, ${ }^{18}$ the prevalence of sleep disorders in childhood has been reported differently depending on the study sample and the treatment providing center. The prevalence of sleep disorders among children of western countries is reported to be $24-40 \%,{ }^{29}$ among Asian children, it is reported to be $70-80 \%,{ }^{30}$ and in Iran, it is reported between 30 and $50 \%$. $^{31,32}$

A study on sleep habits among Brazilian children evaluated these habits based on age, gender, and the school time (mornings, afternoons). The sample of this study included 2482 children between the ages of 7 and 10 years. The results show that $61 \%$ of the children have pre-bedtime rituals; drinking milk before sleeping was higher among seven-year olds compared to others; as age increases, the time lights are on decreases; compared to boys, girls tended to give up their activities and go to bed; compared to children who went to school on afternoons, children who went to school in the mornings had a shorter sleep time, went to bed earlier, and had more naps during the day. Based on these results, it can be concluded that school shifts can have a significant impact on the children's sleep habits. ${ }^{33}$

Another study evaluated the prevalence of sleep problems and sleep habits among elementary school children in Saudi Arabia. The results show that daytime fatigue with $37.5 \%$ had the highest prevalence among sleep problems followed by resistance to going to bed with 
Table 3

Frequency and percentages of current sleep problem and current daytime symptoms.

\begin{tabular}{|c|c|c|c|c|c|}
\hline Variable & & Total & Girls & Boys & $P$-value \\
\hline \multirow[t]{18}{*}{ current sleep problem } & Difficulty breathing when asleep & $58(9.6)$ & $24(7.7)$ & $34(11.7)$ & 0.083 \\
\hline & Snores & $58(9.6)$ & $26(8.3)$ & $32(11)$ & 0.249 \\
\hline & Restless sleep & $46(9.6)$ & $14(4.5)$ & $32(11)$ & 0.002 \\
\hline & Sweating when sleeping & $82(13.5)$ & $38(12.2)$ & $44(15.2)$ & 0.268 \\
\hline & Daytime sleepiness & $50(8.3)$ & $22(7.1)$ & $28(9.7)$ & 0.246 \\
\hline & Poor appetite & $128(21.1)$ & $74(23.7)$ & $54(18.6)$ & 0.138 \\
\hline & Nightmares & $48(7.9)$ & $18(5.8)$ & $30(10.3)$ & 0.036 \\
\hline & Sleepwalking & $16(2.6)$ & $10(3.2)$ & $6(2.1)$ & 0.395 \\
\hline & Sleep talking & 48 (7.9) & $26(8.3)$ & $22(7.6)$ & 0.775 \\
\hline & Screaming in his/her sleep & $52(8.6)$ & $22(7.1)$ & $30(10.3)$ & 0.156 \\
\hline & Kicks legs in sleep & $108(17.8)$ & $58(18.6)$ & $50(17.2)$ & 0.643 \\
\hline & Wakes up at night & $20(3.3)$ & $14(4.5)$ & $6(2.1)$ & 0.105 \\
\hline & Gets out of bed at night & $100(16.5)$ & $54(17.3)$ & $46(15.9)$ & 0.663 \\
\hline & Trouble staying in his/her bed & $30(5)$ & $14(4.5)$ & $16(5.5)$ & 0.520 \\
\hline & Resists going to bed at bedtime & $94(15.5)$ & $44(14.1)$ & $50(17.2)$ & 0.257 \\
\hline & Grinds his/her teeth & $56(9.2)$ & $32(10.3)$ & $2(7.6)$ & 0.275 \\
\hline & Uncomfortable feeling in his/her legs; creepy-crawly feeling & $40(6.6)$ & $18(5.8)$ & $22(7.6)$ & 0.345 \\
\hline & Wets bed & $16(2.6)$ & $2(0.6)$ & $14(4.8)$ & 0.001 \\
\hline \multirow[t]{7}{*}{ current daytime symptoms } & Trouble getting up in the morning & $166(27.4)$ & $78(25)$ & $88(30.3)$ & 0.116 \\
\hline & Falls asleep in school & $50(8.3)$ & $16(5.1)$ & $34(11.7)$ & 0.003 \\
\hline & Naps after school & $210(34.7)$ & $108(34.6)$ & $100(34.5)$ & 0.924 \\
\hline & Daytime sleepiness & $28(4.6)$ & $14(4.5)$ & $14(4.8)$ & 0.812 \\
\hline & Feels weak or loses control of his/her muscles with strong emotions & $22(3.6)$ & $12(3.8)$ & $10(3.4)$ & 0.798 \\
\hline & Reports unable to move when falling asleep or upon waking & $22(3.6)$ & $6(1.9)$ & $16(5.5)$ & 0.018 \\
\hline & Sees frightening visual images before falling asleep or upon waking & $70(11.6)$ & $32(10.3)$ & $38(13.1)$ & 0.260 \\
\hline
\end{tabular}

$26.2 \%$, and difficulty with waking up in the morning with $11.8 \%$. Sleeping with the parents was reported among $12.4 \%$ of the participants (this was 39\% for our study) and taking naps during the day was reported for $40.8 \%$ of the participants. ${ }^{34}$ In our study, $56 \%$ of the participants reported taking naps during the day. Compared to the study in Saudi Arabia, the participants in our study have an undesirable state.

Another study evaluated sleep habits among school children in Texas, U.S. The results show that fear of sleep is prevalent among $43 \%$ of the elementary school participants (which was $7.9 \%$ in our study) and resistance to going to bed, delay in falling asleep, and staying up late were higher among elementary school children than other groups. The results of this study indicate that with the increase in age, the sleep will become more natural. ${ }^{35}$ Another study evaluated sleep pattern, sleep habits, and sleep problems among elementary school children in Japan. The results show that going to bed is delayed more among older children and they have shorter sleep times. Sleep problems were reported higher for first and second grade students than others. ${ }^{36}$

There is a gap in the literature on children's sleep at the first year of school. There is only one study of this nature in Australia whose results show that $12.8 \%$ of children of this age have sleep problems. The results of this study show that using video and audio devices and the difference between the sleep schedule of school nights and non-school nights are among risk factors of sleep problems at this age range. ${ }^{37}$ Therefore, compared to the results of this study, the Iranian children have more worrisome issues with regards to sleep problems.

Sociocultural factors such as the economic state of the family, environmental stress sources, watching television, and lifestyle, ${ }^{38-43}$ are among factors that can affect children's sleeping patterns. Reducing the
Table 5

The correlations between variables.

\begin{tabular}{llll}
\hline Variable & $\begin{array}{l}\text { Sleep } \\
\text { hygiene }\end{array}$ & $\begin{array}{l}\text { Father's } \\
\text { education }\end{array}$ & $\begin{array}{l}\text { Mother's } \\
\text { education }\end{array}$ \\
\hline Sleep problem & $-0.39^{\mathrm{a}}$ & 0.04 & 0.04 \\
General sleep history & 0.04 & $-0.21^{\mathrm{a}}$ & $-0.22^{\mathrm{a}}$ \\
General sleep problem & $-0.24^{\mathrm{a}}$ & $-0.15^{\mathrm{a}}$ & $-0.10^{\mathrm{b}}$ \\
Current sleep problem & $-0.20^{\mathrm{a}}$ & $-0.10^{\mathrm{b}}$ & $-0.10^{\mathrm{b}}$ \\
Current daytime & $-0.27^{\mathrm{a}}$ & $-0.11^{\mathrm{a}}$ & $-0.10^{\mathrm{b}}$ \\
$\quad$ & & & \\
\hline
\end{tabular}

a Correlation is significant at the 0.01 level (2-tailed).

b Correlation is significant at the 0.05 level (2-tailed).

number of daytime naps among three to five-year-old children is a very important factor which reduces the sleep duration. School time is related to sleep time. ${ }^{44}$ In fact, the day and night sleep rhythm is influenced by the specific time the child goes to school. ${ }^{44}$ The behavioral interactions between the parents and the child and the sleep-awake cycles play an important role in children's sleep problems. While these relations are complex and multi-layered, they should be considered carefully. ${ }^{45}$

In another section, the results of our study show that there is a relationship between sleep hygiene and children's sleep habits. A number of previous studies emphasize the importance of regular sleep scheduling. ${ }^{45-47}$ confirmed by the results of the current study. There have been other studies that mention the role of sleep hygiene among children. ${ }^{38-44}$ Whose results are in line with the results of our study. The results of previous studies show that pre-sleep behaviors such as watching television, using screens, or doing homework can be some of

Table 4

The means and standard deviation at general sleep history.

\begin{tabular}{|c|c|c|c|c|}
\hline Variable & Total & Girls & Boys & $P$-value \\
\hline The child's usual bedtime (On school days ) & $10.22 \pm 0.80$ & $10.20 \pm 0.84$ & $10.24 \pm 0.75$ & 0.562 \\
\hline The child's usual wake time (On school days ) & $6.94 \pm 0.68$ & $7.07 \pm 0.86$ & $6.82 \pm 0.39$ & 0.001 \\
\hline The child's usual bedtime (On weekends) & $10.50 \pm 2.45$ & $10.59 \pm 2.28$ & $10.46 \pm 2.63$ & 0.538 \\
\hline The child's usual wake time (On weekends) & $9.54 \pm 1.12$ & $9.60 \pm 1.04$ & $9.48 \pm 1.19$ & 0.202 \\
\hline The amount of time the child spends in his/her bedroom before going to sleep (minutes) & $17.74 \pm 13.09$ & $14.18 \pm 0.82$ & $11.91 \pm 0.70$ & 0.931 \\
\hline
\end{tabular}


Table 6

Binary logistic Regression Models for Child Sleep Problem.

\begin{tabular}{|c|c|c|c|c|c|}
\hline \multirow[t]{3}{*}{ Sleep hygiene } & \multicolumn{5}{|c|}{ Components of BEARS } \\
\hline & Bedtime problems & $\begin{array}{l}\text { Excessive daytime } \\
\text { sleepiness }\end{array}$ & $\begin{array}{l}\text { Awakenings during the } \\
\text { night }\end{array}$ & $\begin{array}{l}\text { Regularity and duration of } \\
\text { sleep }\end{array}$ & $\begin{array}{l}\text { Sleep-disordered } \\
\text { breathing }\end{array}$ \\
\hline & OR $(95 \% \mathrm{CI})$ & OR $(95 \% \mathrm{CI})$ & OR $(95 \% \mathrm{CI})$ & OR $(95 \% \mathrm{CI})$ & OR $(95 \% \mathrm{CI})$ \\
\hline Taking nap & $1(0.80-1.25)$ & $1.45(1.08-1.94)$ & $1.25(0.93-1.68)$ & $0.55(0.43-0.71)$ & $1.21(0.88-1.65)$ \\
\hline Going to bed at different times & $0.98(0.74-1.31)$ & $0.61(0.43-0.88)$ & $1.18(0.83-1.68)$ & $0.96(0.71-1.30)$ & $0.60(0.41-0.89)$ \\
\hline Getting out of bed at different times & $1.21(0.91-1.59)$ & $1.19(0.82-1.73)$ & $0.56(0.40-0.80)$ & $1.39(1.02-1.89)$ & $1.10(0.75-1.64)$ \\
\hline Exercising & $0.93(0.74-1.17)$ & $1.29(0.98-1.71)$ & $0.73(0.54-0.98)$ & $1.31(1.04-1.65)$ & $1.34(1.03-1.72)$ \\
\hline Staying in bed longer & $0.81(0.65-1)$ & $0.86(0.67-1.10)$ & $1.02(0.78-1.34)$ & $1.07(0.84-1.36)$ & $0.96(0.73-1.25)$ \\
\hline Using tobacco or caffeine & $1.31(0.94-1.83)$ & $1.27(0.86-1.84)$ & $0.52(0.36-0.76)$ & $0.80(0.56-1.13)$ & $0.82(0.54-1.24)$ \\
\hline Playing video games & $0.56(0.44-0.72)$ & $0.54(0.41-0.72)$ & $1.01(0.75-1.36)$ & $1.17(0.90-1.55)$ & $1.05(0.74-1.48)$ \\
\hline $\begin{array}{l}\text { Feeling stressed, angry, upset, or } \\
\text { nervous }\end{array}$ & $0.94(0.73-1.22)$ & $1.18(0.84-1.65)$ & $0.71(0.53-0.97)$ & $1.08(0.82-1.42)$ & $1.090(.74-1.59)$ \\
\hline Watching television & $0.94(0.76-1.14)$ & $0.99(0.77-1.28)$ & $0.83(0.64-1.07)$ & $0.66(0.53-0.83)$ & $0.87(0.64-1.16)$ \\
\hline Uncomfortable bed & $0.99(0.76-1.29)$ & $0.85(0.64-1.15)$ & $1.12(0.83-1.51)$ & $0.79(0.60-1.06)$ & $0.88(0.61-1.25)$ \\
\hline Uncomfortable bedroom & $0.99(0.75-1.30)$ & $0.84(0.62-1.13)$ & $1(0.70-1.42)$ & $1.14(0.84-1.55)$ & $1.13(0.76-1.67)$ \\
\hline Doing works & $1.06(.86-1.30)$ & $1.24(0.96-1.60)$ & $1.04(0.79-1.38)$ & $1.40(1.12-1.75)$ & $1.04(0.79-1.35)$ \\
\hline Planning and worrying & $0.49(0.40-0.61)$ & $0.75(0.58-0.98)$ & $0.72(0.55-0.94)$ & $0.49(0.39-0.62)$ & 1.44 (1.16-1.95) \\
\hline Cox \& Snell R Square & 0.18 & 0.12 & 0.11 & 0.21 & 0.05 \\
\hline Nagelkerke R Square & 0.25 & 0.20 & 0.19 & 0.31 & 0.10 \\
\hline
\end{tabular}

Boldface indicates statistically significant $(P<0.05)$.

the factors affecting children's sleep, ${ }^{48,49}$ indicating the role of sleep hygiene. Generally, the results of previous studies show that sleep hygiene among Iranian people is very low, ${ }^{50}$ which can be one of the reasons for the high levels of sleep problems in the selected sample of the current study.

The results of our study show a relatively weak relation between parents' education level and children's sleep patterns and habits. However, there is no significant relationship between parents' education and sleep problems in general. Compared to previous studies, the results of a study show that there is no relationship between parents' education level and children's sleep. ${ }^{51}$ Higher-educated parents have more information about sleep hygiene and they have a higher ability to teach sleep hygiene to their children. On the other hand, it seems that higher-educated parents have more suitable communication strategies for interacting with their children, resulting in their children being more relaxed and calm. Moreover, the child will follow the request of the parents to go to bed early because they want to get the approval of the parents and this is probably how parents affect their children's sleep.

The results of the current study show that for the selected sample, the participants go to bed at 10:22 at school nights and wake up at 6:49. Compared to previous studies, the results of a study show that children in Asian countries go to bed late (about 10:05 p.m.); however, children in Caucasus go to bed earlier (7:45 p.m.), ${ }^{52}$ which is in line with the results of the current study.

Finally, considering the results of the current study and the studies carried out in various countries, it can be argued that sleep patterns, habits, and problems vary based on country and culture. In fact, sleep pattern can be influenced by cultural varieties and the scheduled sleep planned by the family and the culture.

One of the strengths of the current study was the fact that it was carried out on a population with a homogenous culture and language over a specific age range, which is different from previous studies. However, the results are based on the reports of the children's mothers, which is one of the limitations of the current study.

\section{Authors' contributions}

All authors participated in the design of the study and drafted the manuscript and read and approved the final manuscript.

\section{Conflicts of interest}

None of the authors have conflicts of interest to report.

\section{Funding/support}

None.

\section{Appendix A. Supplementary data}

Supplementary data to this article can be found online at https:// doi.org/10.1016/j.cegh.2018.12.007.

\section{References}

1. Galland B, Spruyt K, Dawes P, McDowall PS, Elder D, Schaughency E. Sleep disordered breathing and academic performance: a meta-analysis. Pediatrics 2015;136:e934-e946.

2. Curcio G, Ferrara M, De Gennaro L. Sleep loss, learning capacity and academic performance. Sleep Med Rev. 2006;10:323-337.

3. Edinger JD, Buysse DJ, Deriy L, et al. Quality measures for the care of patients with insomnia. J Clin Sleep Med: JCSM: Off Publ Am Acad Sleep Med. 2015;11:311.

4. Spruyt K, O'brien LM, Cluydts R, Verleye GB, Ferri R. Odds, prevalence and predictors of sleep problems in school-age normal children. J Sleep Res. 2005;14:163-176.

5. Galland BC, Mitchell EA. Helping children sleep. Arch Dis Child. 2010;95:850-853.

6. Muñiz EI. A clinical guide to pediatric sleep: diagnosis and management of sleep problems. J Dev Behav Pediatr. 2012;33:95

7. Nikolopoulou M, St James-Roberts I. Preventing sleeping problems in infants who are at risk of developing them. Arch Dis Child. 2003;88:108-111.

8. Behavioral treatment of bedtime problems and night wakings in infants and young children. Sleep. 2006;29:1263-1276.

9. Sadeh A. Cognitive-behavioral treatment for childhood sleep disorders. Clin Psychol Rev. 2005;25:612-628.

10. Dahl RE, El-Sheikh M. Considering sleep in a family context: introduction to the special issue. J Fam Psychol. 2007;21:1.

11. Stein MA, Mendelsohn J, Obermeyer WH, Amromin J, Benca R. Sleep and behavior problems in school-aged children. Pediatrics. 2001;107 e60-e60.

12. Owens J, Opipari L, Nobile C, Spirito A. Sleep and daytime behavior in children with obstructive sleep apnea and behavioral sleep disorders. Pediatrics. 1998;102:1178-1184.

13. Sadeh A, Gruber R, Raviv A. Sleep, neurobehavioral functioning, and behavior problems in school-age children. Child Dev. 2002;73:405-417.

14. Price AM, Brown JE, Bittman M, Wake M, Quach J, Hiscock H. Children's sleep patterns from 0 to 9 years: Australian population longitudinal study. Arch Dis Child. 2014;99:119-125.

15. Hiscock H, Canterford L, Ukoumunne OC, Wake M. Adverse associations of sleep problems in australian preschoolers: national population study. Pediatrics. 2007;119:86-93.

16. Gregory AM, Rijsdijk FV, Lau JY, Dahl RE, Eley TC. The direction of longitudinal associations between sleep problems and depression symptoms: a study of twins aged 
8 and 10 years. Sleep. 2009;32:189-199.

17. Baglioni C, Battagliese G, Feige B, et al. Insomnia as a predictor of depression: meta-analytic evaluation of longitudinal epidemiological studies. J Affect Disord. 2011;135:10-19.

18. Taras H, Potts-Datema W. Sleep and student performance at school. J Sch Health. 2005;75:248-254.

19. Bos SC, Gomes A, Clemente V, et al. Sleep and behavioral/emotional problems in children: a population-based study. Sleep Med. 2009;10:66-74.

20. Hosseini Araghi M. The Association between Sleep and Obesity and its Impact on Health and Wellbeing. 2014; 2014.

21. Mohammadi M, Ghalebaghi B, Bandi MG, et al. Sleep patterns and sleep problems among preschool and school-aged group children in a primary care setting. Iran $J$ Pediatr (Engl Ed). 2007;17:213-221.

22. Mastin DF, Bryson J, Corwyn R. Assessment of sleep hygiene using the sleep hygiene index. J Behav Med. 2006;29:223-227.

23. Chehri A, Mohammadi H, Negahban S, Khazaie H. Sleep Hygiene Index: Reliability and Validity of the Persian Version in the Male Population. 2016; 2016.

24. Owens JA, Dalzell V. Use of the 'bears'sleep screening tool in a pediatric residents' continuity clinic: a pilot study. Sleep Med. 2005;6:63-69.

25. Spruyt K, Gozal D. Pediatric sleep questionnaires as diagnostic or epidemiological tools: a review of currently available instruments. Sleep Med Rev. 2011;15:19-32.

26. Markovich AN, Gendron MA, Corkum PV. Validating the children's sleep habits questionnaire against polysomnography and actigraphy in school-aged children. Front Psychiatr. 2015;5:188.

27. Kalak N. The Role of Sleep in the Psychological Functioning of Adolescents. 2016; 2016.

28. van Litsenburg RRL, Waumans RC, van den Berg G, Gemke RJ. Sleep habits and sleep disturbances in Dutch children: a population-based study. Eur J Pediatr. 2010;169:1009-1015.

29. Acebo C, Sadeh A, Seifer R, Tzischinsky O, Hafer A, Carskadon MA. Sleep/wake patterns derived from activity monitoring and maternal report for healthy 1 -to 5year-old children. Sleep. 2005;28:1568-1577.

30. Meltzer LJ, Moore M. Sleep disruptions in parents of children and adolescents with chronic illnesses: prevalence, causes, and consequences. J Pediatr Psychol. 2007;33:279-291.

31. N Z, S M, K F. The prevalence of sleep disorders in 1 to 2 year infants referring to health centers in yazd. Toloo-e-behdasht. 2010;8:39-46.

32. P L, K A, S M. Epidemiology of sleep disorders among primary school students in tehran. Andishe VA Raftar. 2004;10:50-58.

33. Silva TA, Carvalho LB, Silva L, et al. Sleep habits and starting time to school in brazilian children. Arquivos de Neuro-psiquiatria. 2005;63:402-406.

34. Hammam AB, AlFaris E, Shaikh S, Saeed AB. Prevalence of sleep problems and habits in a sample of saudi primary school children. Ann Saudi Med. 2006;26:7.

35. Surani S, Hesselbacher S, Surani S, et al. Sleep habits of elementary and middle school children in south Texas. Sleep Disord. 2015;2015.

36. Iwadare Y, Kamei Y, Oiji A, et al. Study of the sleep patterns, sleep habits, and sleep problems in Japanese elementary school children using the cshq-j. Kitasato Med $J$. 2013;43:31-37.

37. Uebergang LK, Arnup SJ, Hiscock H, Care E, Quach J. Sleep problems in the first year of elementary school: the role of sleep hygiene, gender and socioeconomic status. Sleep Health. J Nat Sleep Found. 2017;3:142-147.

38. Cain N, Gradisar M. Electronic media use and sleep in school-aged children and adolescents: a review. Sleep Med. 2010;11:735-742.

39. Cunningham TJ, Ford ES, Chapman DP, Liu Y, Croft JB. Independent and joint associations of race/ethnicity and educational attainment with sleep-related symptoms in a population-based us sample. Prev Med. 2015;77:99-105.

40. Finkelstein DM, Kubzansky LD, Capitman J, Goodman E. Socioeconomic differences in adolescent stress: the role of psychological resources. $J$ Adolesc Health. 2007;40:127-134.

41. Grandner MA, Patel NP, Gehrman PR, et al. Who gets the best sleep? Ethnic and socioeconomic factors related to sleep complaints. Sleep Med. 2010;11:470-478.

42. Owens JA. Introduction: culture and sleep in children. Pediatrics. 2005;115:201-203.

43. Stronks K, Van de Mheen H, Looman CW, Mackenbach JP. The importance of psychosocial stressors for socio-economic inequalities in perceived health. Soc Sci Med. 1998;46:611-623.

44. Sadeh A, Tikotzky L, Scher A. Parenting and infant sleep. Sleep Med Rev. 2010;14:89-96.

45. Beltramini AU, Hertzig ME. Sleep and bedtime behavior in preschool-aged children. Pediatrics. 1983;71:153-158.

46. Biggs SN, Lushington K, van den Heuvel CJ, Martin AJ, Kennedy JD. Inconsistent sleep schedules and daytime behavioral difficulties in school-aged children. Sleep Med. 2011;12:780-786.

47. Pesonen A-K, Räikkönen K, Paavonen EJ, et al. Sleep duration and regularity are associated with behavioral problems in 8-year-old children. Int $J$ Behav Med. 2010;17:298-305.

48. Cespedes EM, Gillman MW, Kleinman K, Rifas-Shiman SL, Redline S, Taveras EM Television viewing, bedroom television, and sleep duration from infancy to midchildhood. Pediatrics. 2014;133:e1163-e1171.

49. Foley LS, Maddison R, Jiang Y, Marsh S, Olds T, Ridley K. Presleep activities and time of sleep onset in children. Pediatrics. 2013;131:276-282.

50. Khazaie H, Chehri A, Sadeghi K, Heydarpour F, Soleimani A, Rezaei Z. Sleep hygiene pattern and behaviors and related factors among general population in west of Iran. Global J Health Sci. 2016;8:114-120.

51. Hense S, Barba G, Pohlabeln H, et al. Factors that influence weekday sleep duration in european children. Sleep. 2011;34:633-639.

52. Mindell JA, Sadeh A, Kwon R, Goh DY. Cross-cultural differences in the sleep of preschool children. Sleep Med. 2013;14:1283-1289. 